\title{
A Computational Study of Internal Flows in a Heated Water-Oil Emulsion Droplet
}

\author{
Jaeheon Sim, ${ }^{1}$ Hong G. $\mathrm{Im}^{2}$, and Suk Ho Chung ${ }^{3}$ \\ King Abdullah University of Science and Technology, Thuwal 23955, Saudi Arabia
}

\begin{abstract}
The vaporization characteristics of water-oil emulsion droplets are investigated by high fidelity computational simulations. One of the key objectives is to identify the physical mechanism for the experimentally observed behavior that the component in the dispersed micro-droplets always vaporizes first, for both oil-in-water and water-in-oil emulsion droplets. The mechanism of this phenomenon has not been clearly understood. In this study, an Eulerian-Lagrangian method was implemented with a temperature-dependent surface tension model and a dynamic adaptive mesh refinement in order to effectively capture the thermo-capillary effect of a micro-droplet in an emulsion droplet efficiently. It is found that the temperature difference in an emulsion droplet creates a surface tension gradient along the micro-droplet surface, inducing surface movement. Subsequently, the outer shear flow and internal flow circulation inside the droplet, referred to as the Marangoni convection, are created. The present study confirms that the Marangoni effect can be sufficiently large to drive the micro-droplets to the emulsion droplet surface at higher temperature, for both water-in-oil and oil-and-water emulsion droplets. A further parametric study with different micro-droplet sizes and temperature gradients demonstrates that larger micro-droplets move faster with larger temperature gradient. The oil micro-droplet in oil-in-water emulsion droplets moves faster due to large temperature gradients by smaller thermal conductivity.
\end{abstract}

\section{Introduction}

$\mathrm{T}$

HE vaporization and combustion characteristics of emulsion fuel droplets have attracted fundamental research interests due to its potential benefits in combustion efficiency and environment. It is known that the emulsion fuel combustion can experience a secondary atomization by micro-explosion, followed by a rapid explosive evaporation from the interior droplet, thus improving combustion efficiency with enhanced air-fuel mixing and reduced total combustion time. Furthermore, the $\mathrm{OH}$ radical production and reduced flame temperature by addition of water suppresses the production of pollutant emissions such as unburned hydrocarbons, nitric oxides, and soot. ${ }^{1}$

Two limiting fundamental models of water-in-oil emulsion droplets combustion were proposed based on the relative volatilities and concentrations of water and oil, the intensity of internal circulation, and the immiscibility. ${ }^{1,2}$ In the slowest frozen limit, there is no liquid movement inside droplet, thus the droplet composition can be assumed to remain as their initial composition. The only possibility for a given liquid element to vaporize is when the droplet surface has regressed to where it is located. However, in the intensely convective limit, namely the distillation limit, there is an active internal circulation such that the droplet interior can be approximated as being spatially uniform but varying only temporally due to vaporization on surface. ${ }^{2}$

The emulsion droplet levitated on a hot surface by the Leidenfrost phenomenon can be categorized in the distillation limit since there exists a strong inner circulation generated by the shear stress exterted at the droplet surface, especially at the bottom surface, by the vaporizing external vapor film flow. A number of studies have been focused on the micro-explosion itself of emulsion droplets, which causes secondary atomization for improved combustion efficiency. An experimental study by Chung and $\mathrm{Kim}^{3}$ reported that there exists a strong mechanism of transporting micro-droplets to the vaporizing emulsion droplet surface such that the component consisting of the micro-droplets always vaporizes first. However, the mechanism of this phenomenon has not been clearly understood or verified.

\footnotetext{
${ }^{1}$ Post-doctoral fellow, Clean Combustion Research Center, jaeheon.sim@kaust.edu.sa, and AIAA member.

${ }^{2}$ Professor, Clean Combustion Research Center, and AIAA Associate Fellow.

${ }^{3}$ Professor, Clean Combustion Research Center.
} 
In order to investigate the physical mechanism of these phenomena, a high-fidelity computational approach is required due to the difficulties in detailed experimental measurements of small micro-droplets physics in an emulsion droplet. Micro-droplets and emulsion droplets are immisible each other and have different material properties. Therefore multiphase modeling should be considered with moving/deforming-surface tracking and interfacial dynamics modeling including surface tension and steep jump in material properties. Moreover, highresolution grids are needed to capture accurately the complex interfacial dynamics around the small micro-droplet surfaces.

Representing and tracking moving interfaces between different phases is the most critical requirement in multiphase computation because the calculation of the surface tension is directly related to precise interface geometry. Many approaches have been proposed, but they can be categorized in three groups; Eulerian, Lagrangian, and Eulerian-Lagrangian method. ${ }^{4}$ Eulerian methods uses a scalar function in stationary grids to extract interface location via post-processing, and have been commonly used in favor of the simplicity in numerical implementation. Volume-of-fluid ${ }^{5}$ and level-set ${ }^{6}$ techniques are the well-known Eulerian method, but the accuracy of the interface representation is limited due to implicit tracking of the interface via a scalar function in a cell. The Lagrangian method is most accurate because the interface can be represented directly on its body-fitted grid and interfacial dynamics is applied on the exact location. However, maintaining satisfactory grid quality remains a challenge, especially when large deformation or topological changes take place. ${ }^{7}$ As a rational compromise, the EulerianLagrangian method can be considered. It utilizes a separate set of Lagrangian moving meshes representing the interface, which is overlaid on a stationary Eulerian grid to compute the flow fields. ${ }^{8}$ In this approach, the interface can be represented accurately due to explicit interface tracking by surface meshes, and it does not require modifications to the computational grid of the field equations. It is known that the interface tracking and surface tension computations are more accurate and efficient in Eulerian-Lagrangian method than Eulerian method at the same level of grid resolutions. ${ }^{9,} 10$ The immersed boundary method proposed by Peskin is categorized in this method. ${ }^{11}$ Sim et al. implemented succefully this Eulerian-Lagrangin method for modeling of a droplet evaporation by local heating and showing the fuel vapor jet ejection induced by Marangoni effect. ${ }^{12}$

In the present study, a water-oil emulsion droplet on a hot surface was considered. As a simplified numerical modeling, a 2-D cylindrical micro-droplet movement in an emulsion droplet with velocity and temperature gradient was simulated in a 2-D computational doamin by implementing the Eulerian-Lagrangian method ${ }^{10}$ with temperaturedependent surface tension model. The micro-droplet movement mechanism, in which micro-droplets always move to surface and vaporize first, was investigated by analyzing the interfacial dynamics around micro-droplets and parametric studies on the micro-droplet size and temperature gradient were followed. Furthermore, the comparison of micro-droplet physics between water-in-oil and oil-in-water emulsion droplets was conducted.

\section{Numerical Method}

\section{A. Problem configuration}

An example of a water-oil emulsion droplet levitated on a hot surface is shown in Fig. 1(a). The emulsion droplet is heated from the hot surface of bottom and the shear stress exerted at the droplet surface by external gas flow and Marangoni effect creates a strong inner circulation. The immiscible micro-droplets moves along the Hill's vortex in an emulsion droplet.

For a high fidelity modeling on the interfacial dynamics, a simplified model problem to represent the movement of a single micro-droplet in a very small region of $40 \mu \mathrm{m} \times 40 \mu \mathrm{m}$ near an emulsion droplet surface is imagined in Fig. 1(b). The flow fields in an emulsion droplet with Hill's vortex and heat transfer from evaporating surface is simplified by assuming constant velocity- and temperature-gradient. As a preliminary simulation, a 2-D computation with a cylindrical micro-droplet was conducted by implementing the Eulerian-Lagrangian method ${ }^{10}$ with a temperature-dependent surface tension model. The surface temperature is assumed $373.12 \mathrm{~K}$, which is the boiling temperature of water at the given pressure of $0.1 \mathrm{MPa}$. Gravitational acceleration is ignored here because its influence on the micro-droplet can be negligible when the density difference of emulsion droplet components is small. Constant and temperature-dependent surface tension models were compared and three different micro-droplet sizes with a diameter of 1,2 , and $4 \mu \mathrm{m}$ and three different temperature gradients of 500,1000 , and $2000 \mathrm{~K} / \mathrm{mm}$ were simulated as a parametric study. For clear demonstration of the mechanism, both oil-in-water and water-in-oil emulsion droplets are compared. The material properties of decane and water are summarized at the boiling temperature of water under $0.1 \mathrm{MPa}$ in Table I. 


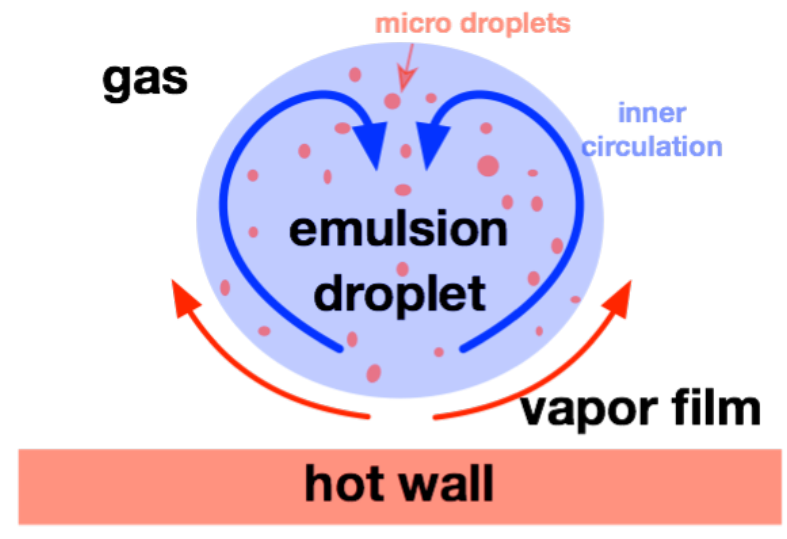

(a)

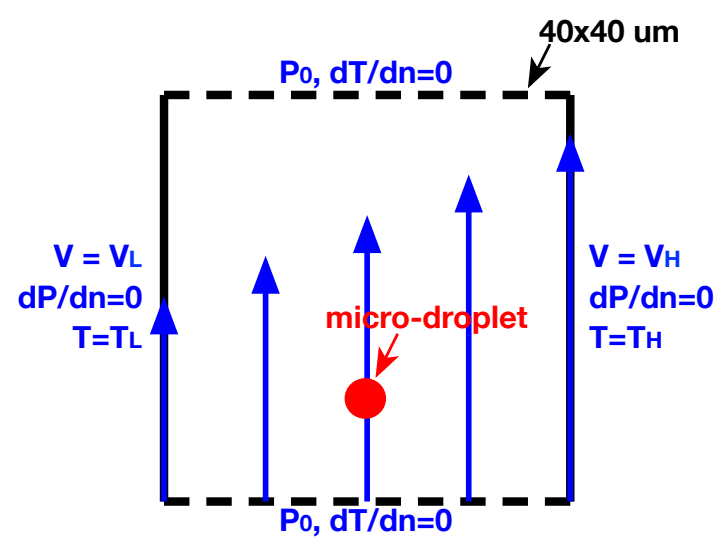

(b)

Figure 1. Problem description of a heated water-oil emulsion droplet. (a) Illustration of an evaporating emulsion droplet levitated on a hot surface. External gas flow and Marangoni effect creates a strong inner circulation. (b) Numerical configuration. A micro-droplet in a flow field with velocity and temperature gradient was numerically simulated with different micro-droplet sizes and temperature gradients.

Table I. Material properties of decane and water at 373.12 K under 0.1 MPa.

\begin{tabular}{lll}
\hline & Decane (liquid) & water \\
\hline \hline Density $\left(\mathrm{kg} / \mathrm{m}^{3}\right)$ & 667.464 & 958.367 \\
Viscosity $(\mathrm{kg} / \mathrm{m} \cdot \mathrm{s})$ & $3.63453 \times 10^{-4}$ & $2.181818 \times 10^{-4}$ \\
Specific heat capacity $(\mathrm{J} / \mathrm{kg} \cdot \mathrm{K})$ & $2.493 \times 10^{3}$ & $4.216 \times 10^{3}$ \\
Thermal conductivity $(\mathrm{W} / \mathrm{m} \cdot \mathrm{K})$ & 0.110943 & 0.679084 \\
Interfacial tension $(\mathrm{N} / \mathrm{m})$ & $0.040\left(\sigma_{T}=\frac{d \sigma}{d T}=-0.00007 \mathrm{~N} / \mathrm{m} \cdot \mathrm{K}\right)$ \\
\hline
\end{tabular}

\section{B. Multiphase modeling: Eulerian-Lagrangian method}

The key objective of this study is to identify the physical mechanism for the experimentally observed behavior ${ }^{3}$ that the component in the dispersed micro-droplets always vaporizes first, for both oil-in-water and water-in-oil emulsion droplets. The evaporation of an emulsion droplet levitated on a hot surface is fundamentally multicomponent, multiphase chemical systems, and has not been studied well with its multiphase interfacial dynamics and heterogeneous combustion characteristics. In this study, a marker-based 3-D Eulerian-Lagrangian method $^{20}$ is implemented with a temperature-dependent surface tension model to predict the thermo-capillary flow in an emulsion droplet. The numerical algorithm utilizes the stationary Eulerian grids to describe the flow field, and moving Lagrangian surface meshes to treat the phase boundaries. The method is chosen in favor of its accurate interface representation and explicit tracking. Capturing the interface geometry is very important since it determines the accuracy of surface tension computation and the associated convective flow and heat transfer.

The interfacial dynamics with discontinuity and surface tension between water and oil is modeled by the continuous interface method (CIM), where the material properties across interfaces are smoothed out by an approximate Dirac-delta function $\left(\delta_{h}\right)^{11}$ within a four-cell width layer, such that a single set of equations for all fluid phases is solved in the entire domain. This approximate Dirac-delta function is used to transfer Lagrangian interfacial quantity to Eulerian fluid quantity, and vice versa. The smoothed fluid properties across interface are computed by: 


$$
\phi=\phi_{2}+\left(\phi_{1}-\phi_{2}\right) I(x)
$$

where the subscript 1 and 2 indicate each phase material, and $\phi$ is the fluid properties such as density $(\rho)$ and the ratio of density to viscosity $(\rho / \mu)$. The indicator function $(I)$ at a given location $(\boldsymbol{x})$ is a scalar function varying from zero to one smoothly across interface and having 0.5 at the interface location. For a better accuracy near boundary, this indicator function is computed as a discrete form of the Heaviside step function $(H)$ by:

$$
I(\boldsymbol{x})=H\left(r=\widehat{\boldsymbol{n}} \cdot\left(\boldsymbol{x}-\boldsymbol{x}_{f}\right)\right)=\int_{-\infty}^{r} \int_{h} \delta_{h}(h) d h,
$$

in which, $\widehat{\boldsymbol{n}}$ is unit vector normal to interface, and subscript $f$ means interface between different phases.

With the help of an approximate Dirac-delta function and indicator function, a single set of conservation equations for mass, momentum, and energy for the entire flow are written as:

$$
\begin{gathered}
\frac{\partial \rho}{\partial t}+\nabla \cdot(\rho \boldsymbol{u})=0 \\
\frac{\partial \rho \boldsymbol{u}}{\partial t}+\nabla \cdot(\rho \boldsymbol{u} \boldsymbol{u})=-\nabla p+\nabla \cdot \mu\left(\nabla \boldsymbol{u}+\nabla^{T} \boldsymbol{u}\right)+\boldsymbol{F}_{f}+\rho \boldsymbol{g} \\
\frac{\partial \rho C_{p} T}{\partial t}+\nabla \cdot\left(\rho C_{p} T \boldsymbol{u}\right)=\nabla \cdot(k \nabla T) .
\end{gathered}
$$

Here, the vector $\boldsymbol{u}$ and $\boldsymbol{g}$ are the velocity vector and the gravitational acceleration vector. The material properties $\rho, \mu, C_{p}$, and $k$ are the density, viscosity, specific heat capacity, and thermal conductivity, respectively. In addition, $t, p$ and $T$ are time, pressure and temperature. The interfacial dynamics are accounted for by the momentum source $\left(\boldsymbol{F}_{f}\right)$ from the phase boundary conditions.

In the normal direction, the phase boundary conditions accounting for the pressure $(p)$ jump and stress tensor $(\tau)$ jump are related to the surface tension $(\sigma)$ and the curvature $(\kappa)$ of the interface by:

$$
\left(p_{2}-p_{1}\right)-\widehat{\boldsymbol{n}} \cdot\left(\tau_{2}-\tau_{1}\right) \cdot \widehat{\boldsymbol{n}}=\sigma \kappa .
$$

From this phase boundary condition, the normal component of the momentum source $\left(\boldsymbol{F}_{f}\right)$ at a given location $(\boldsymbol{x})$ can be computed along phase boundary $(\Gamma)$ directly by:

$$
\boldsymbol{F}_{f}(\boldsymbol{x}) \cdot \widehat{\boldsymbol{n}}=\int_{\Gamma(t)}\left[\sigma\left(T_{f}(\boldsymbol{x})\right) \kappa(\boldsymbol{x}) \cdot \widehat{\boldsymbol{n}}\right] \delta_{h}\left(\boldsymbol{x}-\boldsymbol{x}_{f}\right) d \Gamma
$$

The tangential component of the momentum source $\left(\boldsymbol{F}_{f}\right)$ is incorporated into the Eulerian grids when there exists temperature gradient along the interface, given by:

$$
\boldsymbol{F}_{f}(\boldsymbol{x}) \cdot \hat{\boldsymbol{t}}=\int_{\Gamma(t)}\left[\frac{d \sigma\left(T_{f}(\boldsymbol{x})\right)}{d(\boldsymbol{x} \cdot \hat{\boldsymbol{t}})} \cdot \hat{\boldsymbol{t}}\right] \delta_{h}\left(\boldsymbol{x}-\boldsymbol{x}_{f}\right) d \Gamma .
$$

Surface tension is a given material property, which typically decreases with temperature. A piecewise linear approximation is used to compute accurate surface tension according to the temperature at each location along interface. The surface tension force on a discretized interface can be evaluated by direct curvature computation using surface fitting. However, interpolation-based curvature computation is numerically sensitive to data smoothing, and does not enforce the surface tension conservation of a droplet. Therefore, the computation of line integral form is used with normal and tangential direction of interface elements instead of direct curvature computation because it is much easier, and maintains conservation without expensive curvature computation. ${ }^{8}$

The single set of conservation equations for mass, momentum, and energy for the entire flow in Eqs. (3)-(5) are solved using a projection method, where an intermediate velocity field is computed and projected into a divergencefree space. The convection term is discretized using the third-order ENO scheme in space and the second-order Runge-Kutta integration in time. The central difference scheme along with the Crank-Nicholson method is implemented for the viscous term. The pressure Poisson equation for the velocity correction is solved using the 
conjugate gradient method. The finite volume method is implemented with the staggered grid arrangement, where the face-normal velocity components are stored on the face centers of Cartesian grid cells and other variables (pressure, density, viscosity, etc.) are stored at the cell centers.

To effectively resolve the multiphase flow of multiple length and time scales, an adaptive mesh refinement (AMR) is implemented. The cells are split into four and eight equal sibling cells in 2-D and 3-D, respectively. The grid is represented using unstructured data that connects cells through cell faces. The details of the algorithm can be found in the previous study. ${ }^{10,12}$ The adaptive algorithm is further refined by implementing different AMR levels and layers for the solution-based and geometry-based adaptation. The computational cells are dynamically refined based on the solution of the flow field and the movement of droplet.

\section{Results and Discussion}

As a reference case, a micro-droplet with a diameter of $2 \mu \mathrm{m}$ is simulated under temperature-gradients of $1000 \mathrm{~K} / \mathrm{mm}$. The streamlines of Fig. 2 show that the convective flow is created by the given temperature gradient. The surface tension gradient by temperature difference along the micro-droplet surface induces surface movement, and following outer shear flow and inner Marangoni convection. With this reference case, the comparison of the micro-droplet physics between constant and temperature-dependent surface tension model is conducted under a velocity-gradient of $500 \mathrm{~s}^{-1}$. This velocity gradient is obtained by assuming $10 \mathrm{~cm} / \mathrm{s}$ velocity-difference in $200 \mu \mathrm{m}$ because the center of inner circulation is located around $200 \mu \mathrm{m}$ from the surface with a maximum velocity of $10-20 \mathrm{~cm} / \mathrm{s}$ in our previous research on the droplet evaporation by local heating. ${ }^{12}$ The micro-droplet movements are plottedd in Fig. 3(a). In the temperature-dependent surface tension model, the history of micro-droplet locations in x-direction shows that the micro-droplet moves to cold region by shear flow in the initial transient period, but continues to move to hot surface after transient time. In the unphysical constant surface tension model, the microdroplet doesn't move to either of x-direction and it just moves along the Hill's vortex flow in y-direction. For the temperature-dependent surface model, the micro-droplet moves to hot surface at $0.1-0.2 \mathrm{~mm} / \mathrm{s}$, and this maches well experiment by Chung and Kim. ${ }^{3}$, where it takes several seconds until micro-droplets evaporate first completely. From this comparison, it can be concluded that the surface tension difference by temperature gradient, namely Marangoni effect, may be the key mechanism to transport micro-droplets to hot surface of an emulsion droplet.

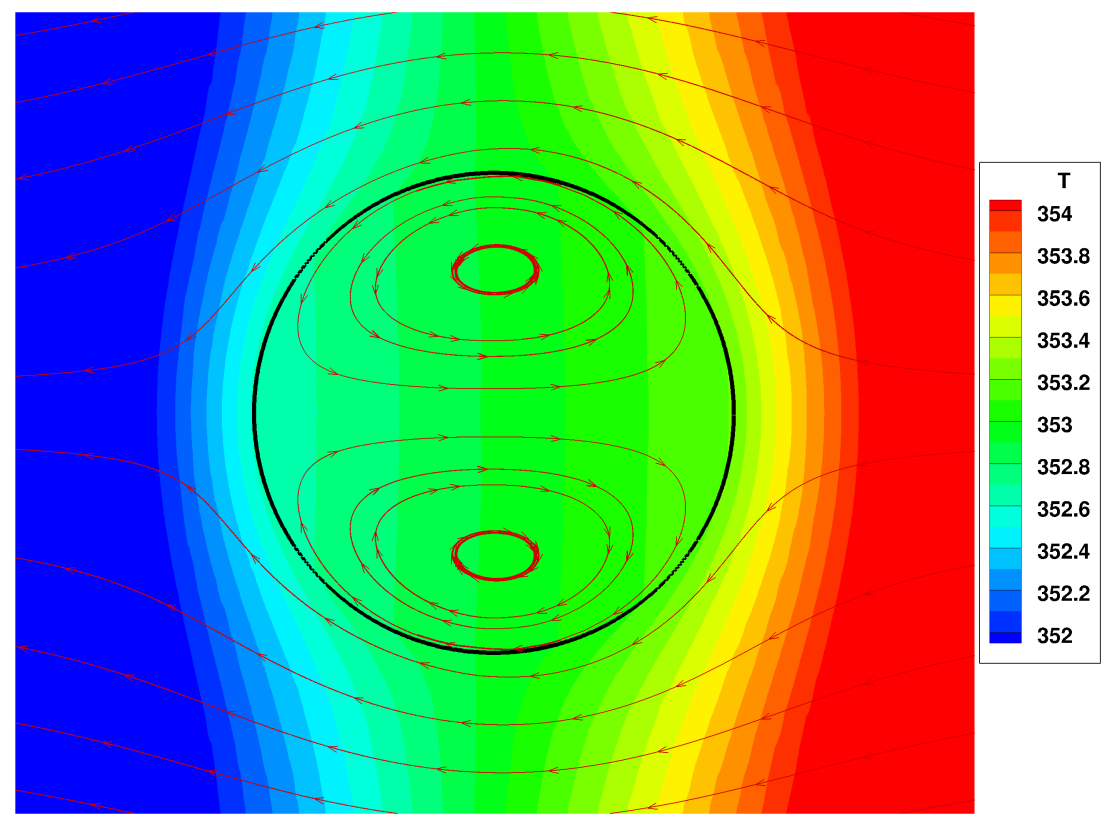

Figure 2. The streamlines and temperature-gradient contour around a micro-droplet in an emulsion droplet. Micro-droplet diameter is $2 \mu \mathrm{m}$ and the temperature gradient in an emulsion droplet is $1000 \mathrm{~K} / \mathrm{mm}$. The streamlines are plotted using relative velocities to the micro-droplet for illustrating flow motions inside and around a micro-droplet separately. 


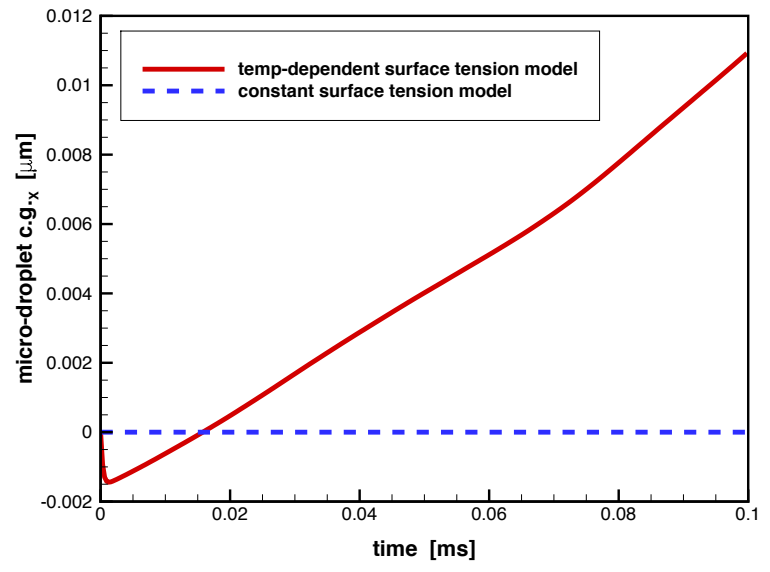

(a)

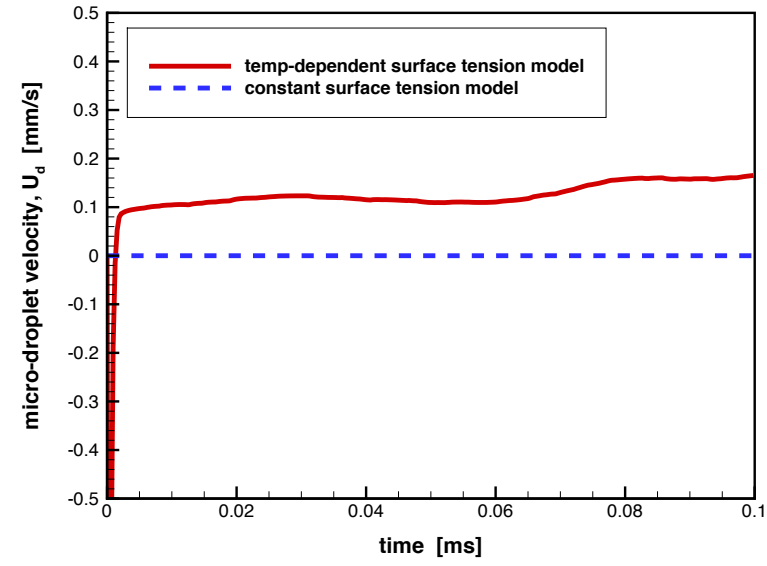

(b)

Figure 3. The comparison of the micro-droplet physics between constant and temperature-dependent surface tension models. Micro-droplet diameter is $1 \mu \mathrm{m}$ under temperature gradient of $1000 \mathrm{~K} / \mathrm{mm}$ and velocitygradient of $500 \mathrm{~s}^{-1}$. (a) The history of micro-droplet locations in $\mathrm{x}$-direction. In a constant surface tension model, the micro-droplet doesn't move to hot surface and it move along the Hill's vortex flow. With the temperature-dependent surface tension model, the micro-droplet moves to cold region in the beginning by shear flow, but moves back to hot surface after transient time. (b) The history of the micro-droplet $x-$ directional velocity. In the temperature-dependent surface tension model, the micro-droplet moves to hot surface at $0.1-0.2 \mathrm{~mm} / \mathrm{s}$.

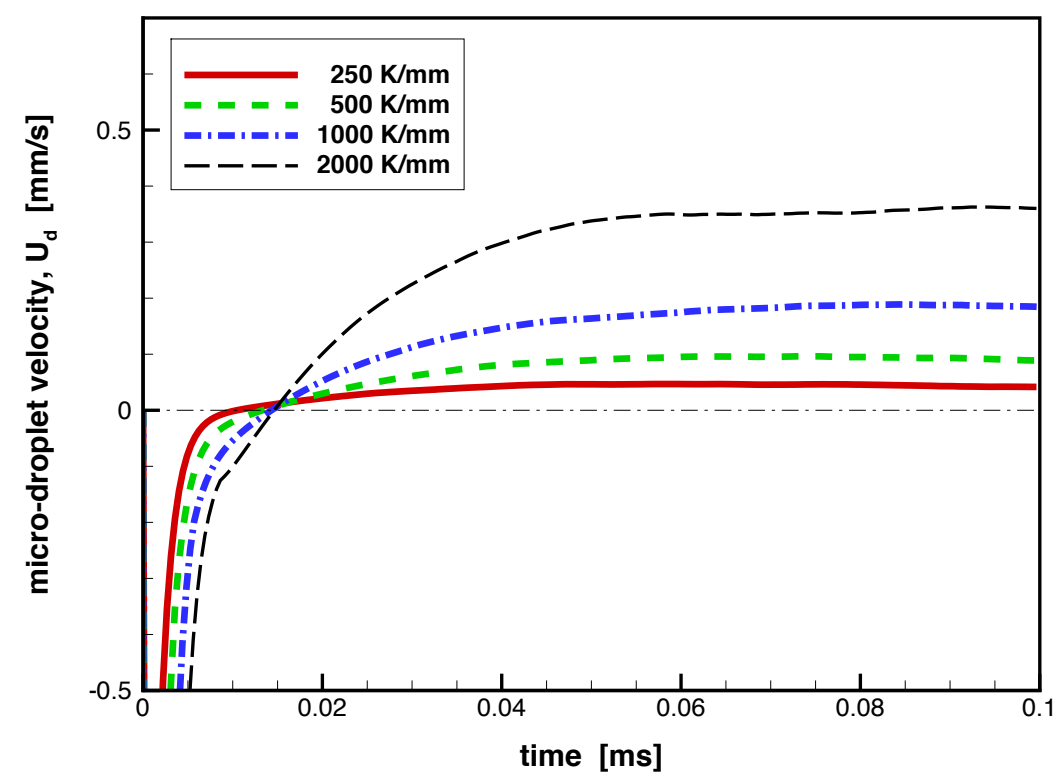

Figure 4. The history of micro-droplet velocities $\left(U_{d}\right)$ with different temperature-gradients from $250 \mathrm{~K} / \mathrm{mm}$ to $2000 \mathrm{~K} / \mathrm{mm}$. Larger temperature-gradient results in faster movement after transient time due to larger Marangoni effect. 


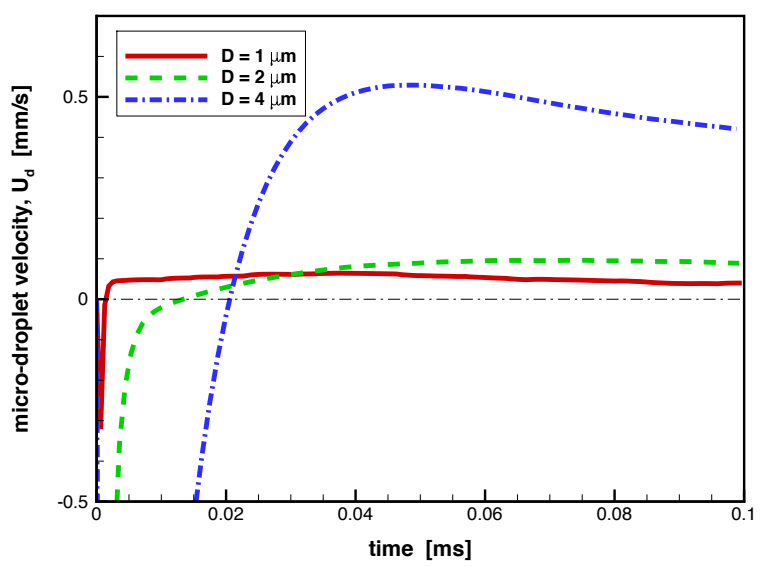

(a)

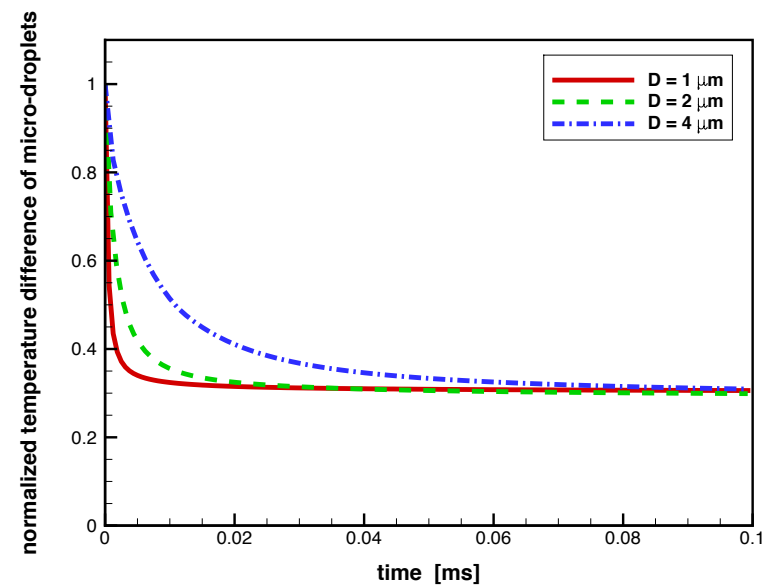

(b)

Figure 5. The influence of micro-droplet sizes on the movement of micro-droplet in an emulsion droplet. Droplet sizes are 1,2 , and $4 \mu \mathrm{m}$. (a) The history of micro-droplet velocities $\left(U_{d}\right)$ with different droplet sizes. Larger droplet moves faster after transient time. (b) Normalized temperature difference of micro-droplets in time. After transient time, the normalized temperature difference is almost same regardless of droplet sizes. The acceleration of micro-droplets is smaller in a larger droplet than a smaller droplet, but longer transient time of larger micro-droplets due to larger volume results in longer acceleration and faster velocities.

The influence of the given temperature gradient $\left(\gamma_{T}=d T / d x\right)$ on the micro-droplet movement was investigated. The x-directional velocity of a micro-droplet $\left(U_{d}\right)$ is plotted with time for different temperature gradients of 250 , 500,1000 , and $2000 \mathrm{~K} / \mathrm{mm}$ in Fig. 4. It shows that the micro-droplet with a diameter of $1 \mu \mathrm{m}$ moves faster as the given temperature gradient increases because the Marangoni effect increases with temperature-gradients. Figure 5 shows the influence of the micro-droplet sizes with a diameter of 1, 2, and $4 \mathrm{~mm}$. The larger micro-droplets move much faster after longer initial transient time. At the same temperature gradient, the surface force is proportional to the surface area of the micro-droplet, and the mass of the micro-droplet is proportional to the volume. Therefore, the acceleration of larger micro-droplet is smaller than that of smaller droplet because the ratio of area to volume decreases with droplet size. This smaller acceleration can be confirmed in the micro-droplet velocity plot in Fig. 5(a), where the velocitiy of smaller droplets changes more rapidly during shorter transient time. However, the temperature gradient in a micro-droplet decreases due to heat transfer as time goes. It decreases much faster for a smaller micro-droplet having smaller volume as shown in the normalized temperature difference plot of Fig. 5(b). Therefore, shorter acceleration period due to faster heat transfer makes smaller micro-droplets move slower.

The physics of micro-droplets are compared between different types of emulsion droplets; water-in-oil emulsion droplet and oil-in-water emulsion droplet. Figure 6 shows that the micro-drople moves faster in the oil-in-water emulsion droplet than it does in the water-in-oil emulsion droplet. The normalized temperature difference plot of Fig. 6(b) shows clearly that the temperature difference of an oil micro-droplet increases in time unlike a water micro-droplet, and results in larger acceleration. Figure 7 shows the temperature contour plots for both cases. The difference of thermal conductivity of water and oil makes the difference in temperature distribution. For the given heat transfer rate, larger thermal conductivity like water results in less temperature gradient in a micro-droplet, and smaller temperature difference moves the micro-droplet slower. 


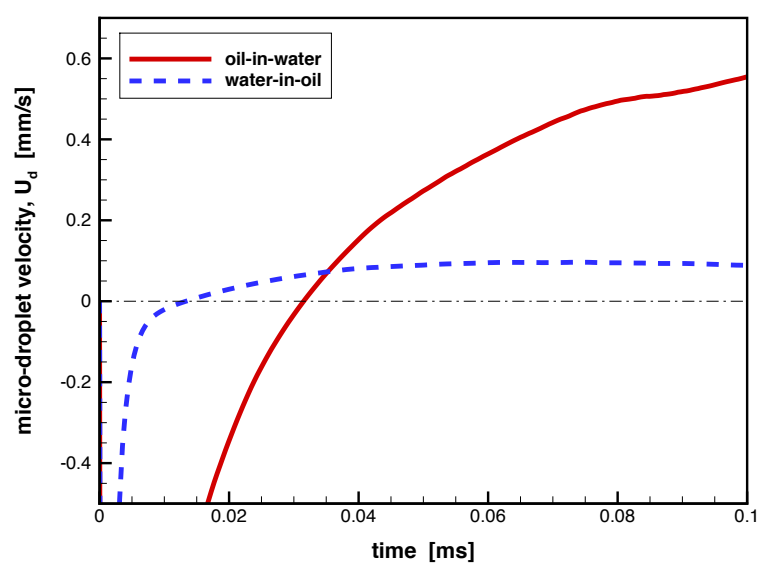

(a)

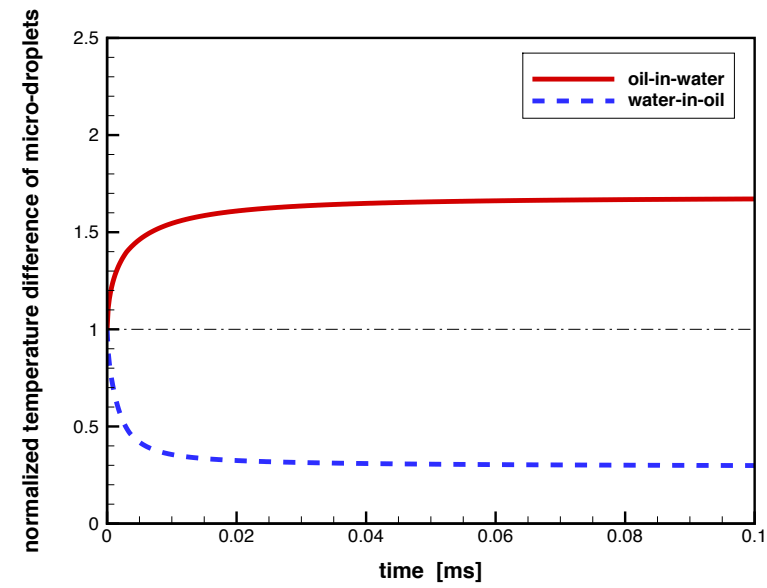

(b)

Figure 6. Comparison of emulsion droplet types; water-in-oil and oil-in-water emulsion droplets. Droplet diameter is $2 \mu \mathrm{m}$ and the temperature gradient is $500 \mathrm{~K} / \mathrm{mm}$. (a) history of micro-droplet velocities. Oil micro-droplet in the oil-in-water emulsion droplet moves faster than water micro-droplet in the water-in-oil emulsion droplet. (b) History of temperature difference of micro-droplet surface. In the water-in-oil droplet, the temperature difference of water droplet surface decreases as heat conducts in a micro-droplet. In the oilin-water emulsion droplet, however, the temperature difference of oil micro-droplet surface increases because the heat conductivity of oil micro-droplet is smaller than that of water.

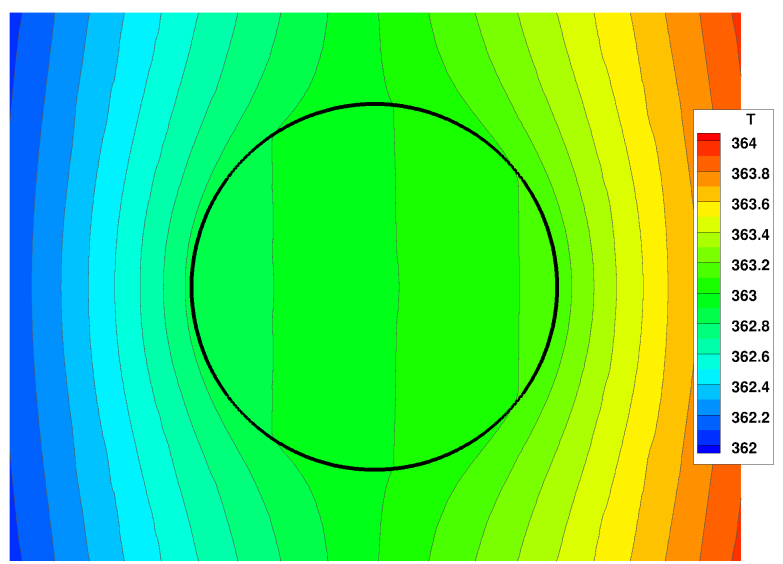

(a)

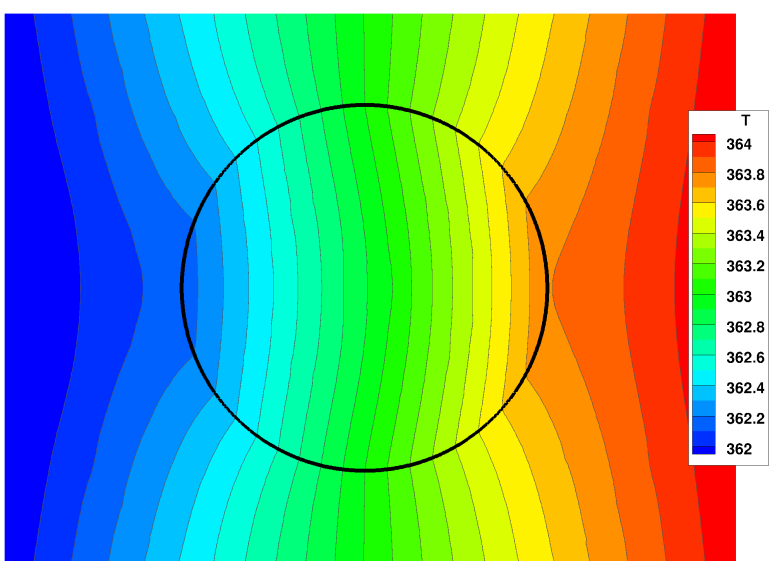

(b)

Figure 7. Comparison of temperature contours of different type of emulsion droplets; water-in-oil and oil-inwater emulsion droplets. Droplet diameter is $2 \mu \mathrm{m}$ and the temperature gradient is $500 \mathrm{~K} / \mathrm{mm}$. (a) Waterin-oil emulsion droplet case. (b) Oil-in-water emulsion droplet case. 


\section{Conclusions}

In this study, the mechanism of the micro-droplet transportation to the hot surface of an evaporating emulsion droplet is investigated. The Eulerian-Lagrangian method ${ }^{10}$ was implemented with a temperature-dependent surface tension model in order to account for the interfacial dynamics of a micro-droplet in an emulsion droplet with Hill's vortex and temperature gradient. The comparison between constant and temperature-dependent surface tension model showed that the surface tension difference by the temperature gradient, namely the Marangoni effect, was sufficient to drive the micro-droplet to the higher temperature region. A further parametric study with different micro-droplet size and temperature gradient showed that larger micro-droplet moves faster with bigger temperaturegradients. The comparison between different types of emulsion droplets showed that the micro-droplet with larger thermal conductivity moves slower due to larger temperature gradient inside a micro-droplet. This explains why oil micro-droplet in a oil-in-water emulsion droplet in general moves faster. The present computational study demonstrated that the Marangoni effect is the key mechanism to transport micro-droplet to the hot surface of emulsion droplets.

\section{References}

${ }^{1}$ Law, C. K., "A Model for the Combustion of Oil/Water Emulsion Droplets," Combustion Science and Technology, Vol. 17, No. 1-2, 1977, pp. 29-38.

${ }^{2}$ Law, C. K., Lee, C. H., and Srinivasan, N., "Combustion characteristics of water-in-oil emulsion droplets," Combustion and Flame, Vol. 37, 1980, pp. $125-143$.

${ }^{3}$ Chung, S. H. and Kim, J. S., "An experiment on vaporization and microexplosion of emulsion fuel droplets on a hot surface," Symposium (International) on Combustion, Vol. 23, No. 1, 1991, pp. 1431 - 1435.

${ }^{4}$ Prosperetti, A. and Tryggvason, G., Computational Methods for Multiphase Flow, Cambridge University Press, New York, 2007.

${ }^{5}$ Hirt, C. W. and Nichols, B. D., "Volume of fluid (VOF) method for the dynamics of free boundaries," Journal of Computational Physics, Vol. 39, No. 1, 1981, pp. 201-225.

${ }^{6}$ Osher, S. and Fedkiw, R., Level Set Methods and Dynamic Implicit Surfaces, Springer, New York, 2002.

${ }^{7}$ Perot, B. and Nallapati, R., "A moving unstructured staggered mesh method for the simulation of incompressible freesurface flows," Journal of Computational Physics, Vol. 184, No. 1, 2003, pp. 192-214.

${ }^{8}$ Uzgoren, E., Sim, J., and Shyy, W., "Marker-based, 3-D Adaptive Cartesian Grid Method for Multiphase Flow around Irregular Geometries," Communications in Computational Physics, Vol. 5, No. 1, 2009, pp. 1-41.

${ }^{9}$ Shyy, W. and Sim, J., "Computational Modeling for Multiphase Flows, Including Microgravity and Space Applications," Encyclopedia of Aerospace Engineering, edited by R. Blockley and W. Shyy, Vol. 1, chap. 51, Wiley, 2011, pp. 597-608.

${ }^{10} \mathrm{Sim}$, J. and Shyy, W., "Interfacial Flow Computations Using Adaptive Eulerian-Lagrangian Method for Spacecraft Applications," International Journal for Numerical Methods in Fluids, Vol. 68, No. 11, 2012, pp. 1438-1456.

${ }^{11}$ Peskin, C. S., "The Immersed Boundary Method," Acta Numerica, Vol. 11, 2003, pp. 479-517.

${ }^{12}$ Sim, J., Im, H. G., and Chung, S. H., "Modeling of Fuel Vapor Jet Eruption Induced by Local Droplet Heating," 52nd Aerospace Sciences Meeting, AIAA2014-1017, American Institute of Aeronautics and Astronautics, 2014. 